\title{
Identification of causative mutations in patients with Leigh syndrome and MERRF by mitochondrial DNA- targeted next-generation sequencing
}

\author{
Hyun Dae Hong', Eunja Kim², Soo Hyun Nam¹, Da Hye Yoo' ${ }^{1}$ Bum Chun Suh ${ }^{3}$, Byung-Ok Choi ${ }^{2, *}$, and Ki Wha Chung ${ }^{1, *}$ \\ 'Department of Biological Sciences, Kongju National University, Gongju, Korea \\ ${ }^{2}$ Department of Neurology and Neuroscience Center, Samsung Medical Center, Sungkyunkwan University School of Medicine, Seoul, Korea \\ ${ }^{3}$ Department of Neurology, Kangbuk Samsung Hospital, Sungkyunkwan University School of Medicine, Seoul, Korea
}

\begin{abstract}
Purpose: Mitochondrial diseases are clinically and genetically heterogeneous disorders, which make their exact diagnosis and classification difficult. The purpose of this study was to identify pathogenic mitochondrial DNA (mtDNA) mutations in 2 Korean families with myoclonic epilepsy with ragged-red fibers (MERRF) and Leigh syndrome, respectively.

Materials and Methods: Whole mtDNAs were sequenced by the method of mtDNA-targeted next-generation sequencing (NGS).

Results: Two causative mtDNA mutations were identified from the NGS data. An m.8344A>G mutation in the tRNA-Lys gene (MT-TK) was detected in a MERRF patient (family ID: MT132), and an m.9176T>C (p.Leu217Pro) mutation in the mitochondrial ATP6 gene (MT-ATP6) was detected in a Leigh syndrome patient (family ID: MT130). Both mutations, which have been reported several times before in affected individuals, were not found in the control samples.

Conclusion: This study suggests that mtDNA-targeted NGS will be helpful for the molecular diagnosis of genetically heterogeneous mitochondrial diseases with complex phenotypes.
\end{abstract}

Key words: Leigh disease, MERRF syndrome, Mitochondrial DNA, MT-ATP6, Mitochondrially encoded tRNA lysine gene, High-throughput nucleotide.

\section{Introduction}

Mitochondrial diseases caused by mitochondrial dysfunction are clinically and genetically a very heterogeneous group of disorders. These diseases primarily result from mutations in the mitochondrial DNA (mtDNA) that lead to oxidative phosphorylation impairment, ATP depletion, cellular dysfunction, and ultimately cell death [1]. Most mitochondrial diseases involve impairment of multiple organs, particularly in tissues with a high energy demand such as nerve and muscle.

Received: 3 June 2015, Revised: 24 July 2015, Accepted: 25 July 2015, Published: 31 December 2015

${ }^{*}$ Co-corresponding authors: Ki Wha Chung, Ph.D.

Department of Biological Sciences, Kongju National University, 56 Gongjudaehak-ro, Gongju 32588, Korea.

Tel: +82-41-850-8506, Fax: +82-41-850-0957, E-mail: kwchung@kongju.ac.kr

Byung-Ok Choi, M.D., Ph.D.

Department of Neurology, Samsung Medical Center, Sungkyunkwan University School of Medicine, 81 Irwon-ro, Gangnam-gu, Seoul 06351, Korea.

Tel: +82-2-3410-1296, Fax: +82-2-3410-0052, E-mail: bochoi77@hanmail.net

Conflict of interest: This study was supported by National Research Foundation (NRF) grants funded by the Korean government, MSIP (2014R1A2A2A01003164 and 2014R1A2A2A01004240), and by the Korean Health Technology R\&D Project, Ministry of Health \& Welfare (HI12C0135 and HI14C3484). The authors declare that they do not have any conflicts of interest.

(c) This is an open-access article distributed under the terms of the Creative Commons Attribution Non-Commercial License (http://creativecommons.org/licenses/by-nc/4.0/) which permits unrestricted non-commercial use, distribution, and reproduction in any medium, provided the original work is properly cited.

(c) Copyright 2015 by the Korean Society of Medical Genetics 
The multi-organ involvement and the overlapping of symptoms among mitochondrial disorders make the exact diagnosis and classification difficult [2-4]. Thus, molecular testing for mtDNA mutations is now an important diagnostic tool for patients with mitochondrial disorders [5].

One of the many mitochondrial diseases, myoclonic epilepsy with ragged-red fibers (MERRF, MIM\# 545000), is caused mainly by maternally inherited mutations in the mitochondrially encoded tRNA lysine gene (MT-TK, MIM\# 590060), such as $m .8344 \mathrm{~A}>\mathrm{G}, \mathrm{m} .8356 \mathrm{~T}>\mathrm{C}$, and $\mathrm{m} .8363 \mathrm{G}>\mathrm{A}$ [6-9]. These point mutations are usually observed to be in the heteroplasmic state [10]. They result in decreased transfer of lysine by mitochondrial tRNA, which consequently disrupts the synthesis of proteins essential for oxidative phosphorylation. In addition to mutations in MT-TK, defects in many other genes have also been reported to present the MERRF phenotype. These include MT-TL1 (mitochondrially encoded tRNA leucine 1, MIM\# 590050) [4,11], MT-TH (mitochondrially encoded tRNA histidine, MIM\# 590040) [12], MT-TS1 (mitochondrially encoded tRNA serine 1, MIM\# 590080) [3], MT-TS2 (MIM\# 590085), MT-TF (mitochondrially encoded tRNA phenylalanine, MIM\# 590070) [13], and MT-ND5 (NADH-ubiquinone oxidoreductase chain 5, MIM\# 516005) [14]. Transfer of mtDNA carrying a tRNA gene mutation to cell lines results in a severe defect in mitochondrial translation in the recipient cells, implying that the tRNA mutation itself is sufficient to cause the disease.

Leigh syndrome (MIM\# 256000) is an early-onset progressive neurodegenerative disorder with a characteristic neuropathology consisting of focal, bilateral lesions in one or more areas of the central nervous system $[15,16]$. Clinical symptoms vary depending on which areas of the central nervous system are involved. Besides its clinical heterogeneity, Leigh syndrome exhibits genetic heterogeneity, with different modes of inheritance: mitochondrial, autosomal recessive, and X-linked recessive $[17,18]$. Mutations in several mtDNA genes are associated with Leigh syndrome, such as MT-FMT (mitochondrially encoded methionyl-tRNA formyltransferase, MIM\# 611766), MT-ATP6 (mitochondrially encoded ATP synthase $F_{0}$ subunit 6, MIM\# 516060), MT-ND5, and MT-ND6 (MIM\# 516006). In particular, $m .8993 \mathrm{~T}>\mathrm{G} / \mathrm{C}$ and $\mathrm{m}$.10158T>C have been frequently reported in Leigh syndrome [19-23].

Ever since the human mtDNA had been completely sequenced [24], several hundreds of different mtDNA mutations have been reported to be associated with various mitochondrial diseases in the Human Mitochondrial Genome Database (MITOMAP; http://mitomap.org/MITOMAP). However, mtDNA analysis studies have not been actively performed in Korean patients with mitochondrial diseases [25-27]. Whole-mtDNA sequencing is usually performed by polymerase chain reaction (PCR) amplification of mtDNA with 46 fragments, with subsequent Sanger sequencing using a mitoSEQr resequencing system (Life Technology, Grand Island, NY, USA). Next-generation sequencing (NGS) technology, which has revolutionized the field of molecular biology, has recently been applied in the diagnosis of mitochondrial diseases $[28,29]$. The NGS technique is now recognized to reduce sequencing cost and analysis time, with high coverage and fidelity, thereby enabling the decoding of a number of human mitochondrial diseases. Calabrese et al. [30] developed an MToolBox pipeline to reconstruct and analyze human mtDNA from high-throughput sequencing data.

In the present study, we analyzed 2 Korean patients with MERRF and Leigh syndromes, respectively, by applying an mtDNA-targeted NGS method into whole-mtDNA sequencing. From the analysis, $2 \mathrm{mtDNA}$ mutations were identified as the underlying causes of each disorder: an m.8344A $>G$ mutation in MT-TK from the MERRF patient, and an m.9176T>C (Leu217Pro) mutation in MT-ATP6 from the Leigh syndrome patient.

\section{Materials and Methods}

\section{Subjects}

This study included mitochondrial diseases from 2 families
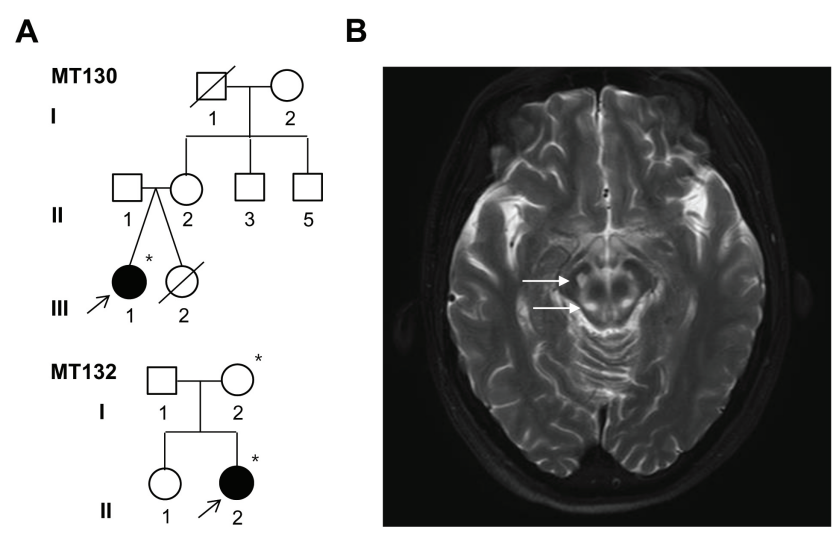

Fig. 1. Pedigrees of 2 patients with mitochondrial disorders and brain magnetic resonance imaging (MRI) of patient 1. (A) Pedigrees of the MT130 and MT132 families. The open symbols represent unaffected individuals and filled symbols represent affected individuals. The probands are indicated by the arrows. Asterisks indicate individuals whose DNAs were used in this study. (B) Brain MRI of patient 1 with the Leigh syndrome. T2 weighted images revealed the hyperintense lesion mainly involving the midbrain level (arrow). These lesions were symmetrical and longitudinally extended from the bilateral caudate nucleus to the dorsal pons area with sparing of the mammillary body. 
of Korean origin: 1 with Leigh syndrome (family ID: MT130) and 1 with MERRF (family ID: MT132) (Fig. 1A). In addition to these 2 families, 300 healthy individuals were examined as the healthy controls. Written informed consent was obtained from all participants according to a protocol approved by the institutional review board for Sungkyunkwan University, Samsung Medical Center (2013-11-057-021).

\section{Clinical assessment}

Clinical information included assessments of age at onset, mental status, cranial nerve function, muscle impairments, sensory loss, and deep tendon reflexes. Age at onset was determined by asking patients what their age was when symptoms first appeared. Neurophysiological studies were carried out on 4 affected individuals ( 3 males and 1 female). Motor and sensory conduction velocities of median, ulnar, peroneal, tibial, and sural nerves were determined, and needle electromyography was performed in the bilateral upper and lower limb muscles. Laboratory tests included levels for creatine kinase, copper, ceruloplasmin, lactic acid, and thiamine.

Brain magnetic resonance images were evaluated using a 1.5T system (Siemens Vision; Siemens, Erlangen, Germany). Whole brains were scanned using a slice thickness of $7 \mathrm{~mm}$ and a 2-mm interslice gap, to produce 16 axial images. The imaging protocol consisted of T2-weighted spin echo (TR/TE=4,700/120 ms), T1weighted spin echo (TR/TE=550/12 ms), and fluid-attenuated inversion recovery ( $\mathrm{TR} / \mathrm{TE}=9,000 / 119 \mathrm{~ms}$; inversion time 2,609 $\mathrm{ms}$ ) images.

\section{DNA purification and determination of mtDNA common deletion}

DNA was extracted from peripheral blood, using the OIAamp DNA blood mini kit (Qiagen, Hilden, Germany). Long-template PCR was performed to determine the common deletion of mtDNA $(8,469-13,447)$, with forward (m.8182-8208) and reverse (m.13981-13957) primer pairs, by using the Expand 20 kb PLUS PCR system (Roche Diagnostics GmbH, Mannheim, Germany). The PCR condition consisted of initial denaturation at $92^{\circ} \mathrm{C}$ for $2 \mathrm{~min}$, followed by 30 cycles of $92^{\circ} \mathrm{C}$ for $10 \mathrm{~s}, 58^{\circ} \mathrm{C}$ for $30 \mathrm{~s}, 68^{\circ} \mathrm{C}$ for $10 \mathrm{~min}$, and a final extension at $68^{\circ} \mathrm{C}$ for $7 \mathrm{~min}$.

\section{Mitochondrial DNA-targeted next-generation sequencing}

The mtDNA-targeted NGS was applied to 2 affected individuals from both families (III-1 in MT130 and II-2 in MT132). The probe design for target capture was performed according to Suredesign wedsite (https://earray.chem.agilent.com/suredesign; Agilent,
Santa Clara, CA, USA), and sequencing was performed with the HiSeq 2000 Genome Analyzer (Illumina, San Diego, CA, USA). The UCSC hg 19 assembly (GRCh37, mtDNA GenBank Accession No. NC_001807.4) was used as the reference sequence for mapping and alignment. All variant calls from whole mtDNA were checked to see whether they were registered in the dbSNP142 (http:// www.ncbi.nIm.nih.gov) and MITOMAP database. All insertion/ deletions (indels) and candidate causative variants were confirmed by Sanger's sequencing method, using the Big Dye Terminator Cycle Sequencing Ready Reaction Kit (Life Technology, Grand Island, NY, USA) and the ABI3130XL Genetic Analyzer (Life Technology). Because the mtDNA sequences from the NGS were primary aligned by the hg 19 assembly (NC_001807.4), variants were compared with the revised Cambridge reference sequence ( $\left.r C R S, N C \_012920.1\right)$, which is commonly used as the reference sequence for mtDNA studies [31].

\section{Determination of heteroplasmic rate}

The heteroplasmic rate was measured by dual methods. First, the rate was determined as the ratio of altered reads per total read depth at the mutation site from the NGS data. Second, PCR-amplified DNA fragments including the mutation site were cloned into the pGEM-T Easy vector (Promega, Madison, WI, USA), which was then used to transform Escherichia coli ( $\mathrm{DH} 5 \alpha)$. Plasmids were isolated from about 100 colonies/mutation, and the mutation rate was then determined by counting the clones that had a mutation.

\section{Haplogrouping and conservation analysis}

The mtDNA haplogroup was determined by using the HaploGrep software based on PhyloTree Build 16 (http:// haplogrep.uibk.ac.at) and the mtDNAmanager (http:// $\mathrm{mtmanager.yonsei.ac.kr)} \mathrm{tool.} \mathrm{Multiple} \mathrm{alignments} \mathrm{of} \mathrm{the} \mathrm{amino}$ acid sequences from different species were performed using the MEGA5 program (ver. 5.05; http://www.megasoftware.net/) by executing the ClustalW algorithm.

\section{Results}

\section{Clinical manifestations}

1) Patient 1

A 21-year-old female (Fig. 1A; MT130, III-1) presented at the emergency room with stupor mentality. She was born at full term from healthy parents, and early milestones were normal. From the age of 14 years, she had difficulty in making friends 
and her mood fluctuated severely. She had acted like a child since 18 years of age. She was admitted to a college 2 years ago but did not adapt well. In addition, she began to eat only what she wanted, such as milk, broccoli, and sweet potatoes. Twelve days before the hospital visit, she had a high fever, vomiting, and diarrhea, and on the visiting day, she could not awake spontaneously. On neurological examination at 21 years, her mental status was stuporous and her orientation to time, place, and person was severely impaired. The blood creatine kinase level was $660 \mathrm{IU} / \mathrm{L}$ and other values, including complete blood count, liver and kidney function test, copper, ceruloplasmin, lactic acid, and thiamine, were normal. Brain magnetic resonance imaging (MRI) revealed high signal intensity on the T2-weighted image in the bilateral paramedian thalamus, midbrain, pons, and paraventricular area (Fig. 1B).

Table 1. Mitochondrial DNA-targeted next-generation sequencing

\begin{tabular}{ll}
\hline \multicolumn{1}{c}{ Item } & \multicolumn{1}{c}{ Information } \\
\hline Target sequence & NC 001807.4 \\
& UCSC hg19 assembly (GRCh37) \\
Coverage & $100 \%$ \\
\hline Average read depth & 6,586 reads \\
Number of SNPS (including indels) $^{a}$ & MT130, 42; MT132, 31 \\
\hline
\end{tabular}

${ }^{a}$ Single nucleotide polymorphism (SNP) numbers were obtained from comparison with revised Cambridge reference sequence (NC_012920.1). Indel, insertion and deletion polymorphism.

\section{2) Patient 2}

A 27-year-old female (Fig. 1A; MT132, II-2) had complaints of ataxic gait and myoclonus. She was born at full term from healthy parents, and early milestones were normal. At 13 years of age, she began to stagger and easily fall, and the symptoms worsened progressively. Myoclonus was elicited by photic stimulus or voluntary movement of the limbs. On neurological examination at 27 years, ataxia, disability of tandem gait, bilateral hand tremor, and intermittent myoclonus were observed. Her muscle strengths and sensory functions were normal and deep tendon reflexes were normoreflexive. Electrophysiological studies, including nerve conduction study and electromyography, did not reveal any abnormal findings.

\section{Identification of causative mutations by mtDNA-targeted next-generation sequencing}

To identify genetic causes, whole-mtDNA-targeted NGS was performed using mtDNA from the 2 patients described above (Table 1). The whole target was completely covered (mtDNA coverage: 100.0\%) with an average read depth of 6,586X. MT130 (III-1) and MT132 (II-2) showed 42 and 31 variants (including indels) in total, respectively, when their mtDNA sequences were compared with the rCRS mtDNA [31]. All the sequencing variants are shown in Table 2. The haplogroups of the $2 \mathrm{mtDNA}$ sequences were estimated to be D5a 1 (MT130) and N9a5 (MT132), respectively.

Table 2. Variants identified from mtDNA-targeted next-generation sequencing in two patients

\begin{tabular}{|c|c|c|c|c|c|c|}
\hline \multirow{2}{*}{ Locus/gene } & \multicolumn{2}{|c|}{ Mutation } & \multirow{2}{*}{$\begin{array}{c}\text { MT130 } \\
(\text { III-1) }\end{array}$} & \multirow{2}{*}{$\begin{array}{c}\text { MT132 } \\
(\text { II-2) }\end{array}$} & \multicolumn{2}{|c|}{ Report } \\
\hline & Nucleotide & Amino acid & & & dbSNP142 & MITOMAP \\
\hline \multirow[t]{8}{*}{ D-loop } & $m .68 G>A$ & - & $A$ & G & - & Pol \\
\hline & $\mathrm{m} .73 \mathrm{~A}>\mathrm{G}$ & - & G & G & rs3087742 & Pol \\
\hline & $\mathrm{m} .150 \mathrm{C}>\mathrm{T}$ & - & $\mathrm{T}$ & $\mathrm{T}$ & rs62581312 & Pol \\
\hline & $\mathrm{m} .263 \mathrm{~A}>\mathrm{G}$ & - & G & G & rs2853515 & Pol \\
\hline & m.303.1insC & - & - & InsC & rs369786048 & Pol \\
\hline & $\mathrm{m} .310 \mathrm{~T}>\mathrm{C}$ & - & $T$ & C & - & Pol \\
\hline & m.315.1insC & - & InsC & InsC & - & Pol \\
\hline & m.489T>C & - & C & $\mathrm{T}$ & rs28625645 & Pol \\
\hline \multirow[t]{4}{*}{$M T-R N R 1$} & m.750A $>G$ & - & G & G & rs2853518 & Pol \\
\hline & $\mathrm{m} .752 \mathrm{C}>\mathrm{T}$ & - & $T$ & C & rs2853518 & Pol \\
\hline & m.1107T>C & - & C & $\mathrm{T}$ & rs111033323 & Pol \\
\hline & $\mathrm{m} .1438 \mathrm{~A}>\mathrm{G}$ & - & G & G & rs2001030 & Pol \\
\hline MT-RNR2 & m.2706A $>G$ & - & $G$ & $G$ & rs2854128 & Pol \\
\hline \multirow[t]{3}{*}{ MT-ND1 } & m.3496G $>T$ & p.Ala64Ser & $\mathrm{T}$ & G & - & Pol/LHON \\
\hline & m.3729A $>G$ & Syn & $A$ & G & - & Pol \\
\hline & m. $4136 A>G$ & p.Tyr277Cys & G & A & rs199476121 & Pol/LHON \\
\hline
\end{tabular}


Table 2. Continued

\begin{tabular}{|c|c|c|c|c|c|c|}
\hline \multirow{2}{*}{ Locus/gene } & \multicolumn{2}{|c|}{ Mutation } & \multirow{2}{*}{$\begin{array}{c}\text { MT130 } \\
(\text { III-1) }\end{array}$} & \multirow{2}{*}{$\begin{array}{c}\text { MT132 } \\
(\text { II-2) }\end{array}$} & \multicolumn{2}{|c|}{ Report } \\
\hline & Nucleotide & Amino acid & & & dbSNP142 & MITOMAP \\
\hline \multirow[t]{7}{*}{ MT-ND2 } & m.4688T $>C$ & Syn & $T$ & C & - & Pol \\
\hline & m.4769A>G & Syn & G & G & rs3021086 & Pol \\
\hline & m.4883C>T & Syn & $\mathrm{T}$ & C & rs200763872 & Pol \\
\hline & m.5178C $>A$ & p.Leu237Met & $A$ & C & rs28357984 & Pol \\
\hline & $\mathrm{m} .5231 \mathrm{G}>\mathrm{A}$ & Syn & $G$ & $A$ & rs371345850 & Pol \\
\hline & $\mathrm{m} .5301 \mathrm{~A}>\mathrm{G}$ & p.lle278Val & $G$ & $A$ & rs199794187 & Pol \\
\hline & $m .5417 G>A$ & Syn & $G$ & $A$ & rs386828968 & Pol \\
\hline MT-C01 & $\mathrm{m} .7028 \mathrm{C}>\mathrm{T}$ & Syn & $T$ & $T$ & rs2015062 & Pol \\
\hline MT-TK & $\mathrm{m} .8344 A>G$ & - & A & G & rs118192098 & MERRF \\
\hline \multirow[t]{4}{*}{ MT-ATPG } & $m .8701 A>G$ & p.Thr59Ala & G & $A$ & rs2000975 & Pol \\
\hline & $m .8860 A>G$ & p.Thr112Ala & G & G & rs2001031 & Pol \\
\hline & m.9176T>C & p.Leu217Pro & C & $T$ & rs199476135 & LS \\
\hline & m. $9180 A>G$ & Syn & G & $A$ & rs2298011 & Pol \\
\hline MT-CO3 & m.9540T>C & Syn & C & $\mathrm{T}$ & rs2248727 & Pol \\
\hline \multirow[t]{3}{*}{ MT-ND3 } & m.10397A $>G$ & Syn & $G$ & $A$ & - & Pol \\
\hline & $m .10398 A>G$ & p.Thr114Ala & G & $A$ & rs2853826 & Pol \\
\hline & $m .10400 C>T$ & Syn & $\mathrm{T}$ & C & rs28358278 & Pol \\
\hline \multirow[t]{5}{*}{ MT-ND4 } & m.10873T>C & Syn & C & $\mathrm{T}$ & rs2857284 & Pol \\
\hline & m.11719G $>A$ & Syn & $A$ & $A$ & rs2853495 & Pol \\
\hline & m.11944T>C & Syn & C & $\mathrm{T}$ & rs3087901 & Pol \\
\hline & m.12026A $>G$ & p.lle423Val & G & $A$ & rs202136725 & Pol/DM \\
\hline & m.12040A $>G$ & Syn & G & $A$ & - & Pol \\
\hline \multirow[t]{4}{*}{ MT-ND5 } & m.12705C>T & Syn & $\mathrm{T}$ & C & rs267606893 & Pol \\
\hline & m.12358A $>G$ & p.Thr8Ala & $A$ & G & rs201027657 & Pol \\
\hline & $m .12372 G>A$ & Syn & G & $A$ & rs2853499 & Pol \\
\hline & $m .13708 G>A$ & p.Ala458Thr & $A$ & $A$ & rs28359178 & $\mathrm{Pol} / \mathrm{LHON}$ \\
\hline \multirow[t]{6}{*}{$M T-C Y B$} & m.14766C>T & p.Thr7lle & $\mathrm{T}$ & $\mathrm{T}$ & rs28359178 & Pol \\
\hline & m.14783T>C & Syn & C & $\mathrm{T}$ & rs527236042 & Pol \\
\hline & $m .15043 G>A$ & Syn & $A$ & G & rs527236043 & Pol \\
\hline & m.15301G $>A$ & Syn & $A$ & G & rs527236045 & Pol \\
\hline & m.15326A $>G$ & p.Thr194Ala & G & G & rs2853508 & Pol \\
\hline & m.15883G $>A$ & Syn & G & $A$ & - & Pol \\
\hline \multirow[t]{10}{*}{ D-Loop } & m.16046T>C & - & $\mathrm{T}$ & C & - & Pol \\
\hline & $\mathrm{m} .16172 \mathrm{~T}>\mathrm{C}$ & - & $\mathrm{T}$ & C & rs2853508 & Pol \\
\hline & m.16182A $>C$ & - & C & C & - & Pol \\
\hline & m.16209T>C & - & $\mathrm{T}$ & C & rs386829278 & Pol \\
\hline & m.16223C>T & - & $\mathrm{T}$ & $\mathrm{T}$ & rs2853513 & Pol \\
\hline & m.16257C>A & - & C & $A$ & rs376682258 & Pol \\
\hline & m.16261C>T & - & C & $\mathrm{T}$ & rs138126107 & Pol \\
\hline & m.16309A $>G$ & - & $A$ & G & rs373517769 & Pol \\
\hline & m.16390G >A & - & $A$ & $A$ & rs41378955 & Pol \\
\hline & m.16519T>C & - & C & C & rs3937033 & Pol \\
\hline
\end{tabular}

MITOMAP: Human Mitochondrial Genome Database (http://mitomap.org/MITOMAP).

Syn, synonymous mutation; Pol, polymorphic; LHON, Leber's hereditary optic neuropathy; MERRF, myoclonic epilepsy and ragged-red fibers; DM, diabetes mellitus; LS, Leigh syndrome. 
A

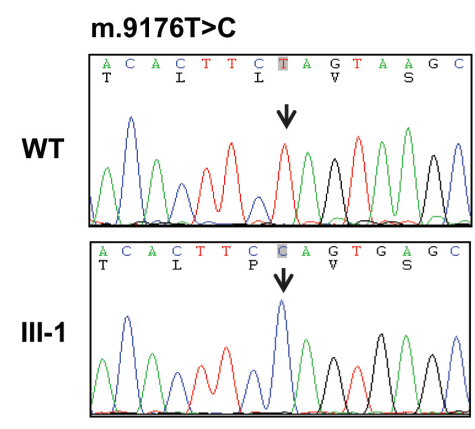

m.8344A>G

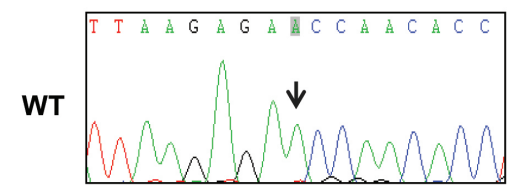

II-2

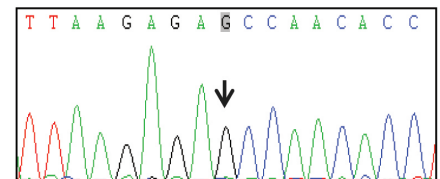

$1-2$

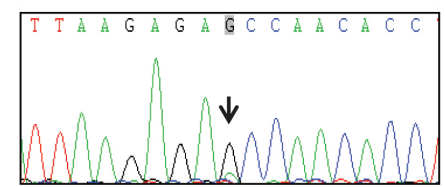

B

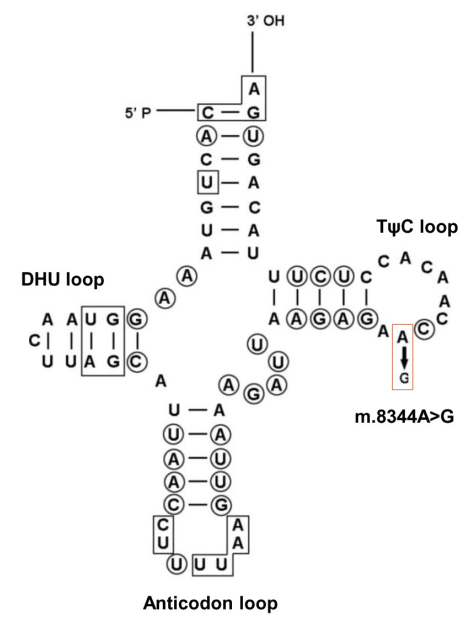

C

p.Leu217Pro

\begin{tabular}{|c|c|c|c|c|c|c|c|c|c|c|c|c|c|c|}
\hline Human & $Q$ & A & Y & V & $F$ & $\mathrm{~T}$ & $\mathrm{~L}$ & L & V & $S$ & L & Y & L & $\mathrm{H}$ \\
\hline Rat & Q & A & Y & V & $\mathrm{F}$ & $\mathrm{T}$ & L & L & V & S & L & $Y$ & L & $\mathrm{H}$ \\
\hline Bov & $Q$ & A & $Y$ & V & $F$ & $\mathrm{~T}$ & L & L & V & S & L & $Y$ & L & $\mathrm{H}$ \\
\hline She & $Q$ & A & Y & V & F & $\mathrm{T}$ & L & L & V & S & L & $Y$ & L & $\mathrm{H}$ \\
\hline Xenopus & $Q$ & A & $Y$ & V & $\mathrm{F}$ & V & L & $\mathrm{L}$ & $\mathrm{L}$ & S & L & $Y$ & L & $Q$ \\
\hline
\end{tabular}

m.8344A>G

\begin{tabular}{|c|c|c|c|c|c|c|c|c|c|c|c|c|c|c|c|}
\hline Human & $T$ & A & A & G & A & G & A & A & C & C & A & A & C & A & \\
\hline Rat & T & & A & G & A & G & & A & C & 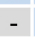 & A & A & C & A & \\
\hline Mouse & $T$ & - & A & G & A & G & - & A & C & C & $\mathrm{T}$ & $\mathrm{T}$ & A & A & \\
\hline Bovine & $\mathrm{T}$ & G & A & G & A & G & - & - & C & C & A & $T$ & A & $\mathrm{T}$ & \\
\hline Xenopus & $T$ & G & - & G & $T$ & G & - & A & C & $\mathrm{T}$ & C & C & C & A & \\
\hline
\end{tabular}

Fig. 2. Identification of causative mutations in mtDNA from 2 mitochondrial patients. (A) Sequencing chromatograms of two mutations, m.8344A>G in the myoclonic epilepsy with ragged-red fibers (MERRF) patient (MT132) and m.9176T>C in the Leigh syndrome patient. The unaffected individuals (proband's mother: I-2 in MT132) shows heteroplasmy for the m.8344A $>$ G. (B) Diagrammatic representation of tRNA-Lys. The position of the A-to- $\mathrm{G}$ base substitution $(\mathrm{m} .8344 \mathrm{~A}>\mathrm{G})$ in the pseudouridine $(\mathrm{T} \psi \mathrm{C})$ loop is indicated by a red box. The nucleotides within boxes are conserved in mammals (human, cow, mouse, rat, and hamster), whereas the nucleotides within circles are conserved throughout a wider range of species including chicken, Xenopus, cod, honeybee, and fruit fly. (C) Conservation analysis of the two mutation sites.

From the screen, 2 causative mtDNA mutations were identified in the examined families. An m.8344A>G mutation in MT-TK was detected in the MERRF patient (family ID: MT132), and an m.9176T>C (p.Leu217Pro) mutation in MT-ATP6 was detected in the Leigh syndrome patient (family ID: MT130). Both mutations were found in the affected probands as the homoplasmy, whereas m.8344A $>G$ was found in the patient's mother (I-2) as a heteroplasmic state with an approximate mutation rate of 80\% (Fig. 2A). Both mutations, which have been reported several times in MERRF and Leigh syndrome patients [32-34], were not found in the 300 control samples. The $\mathrm{m} .8344 \mathrm{~A}>\mathrm{G}$ mutation was located in the less conserved region, but it was within the pseudouridine arm which plays an important role on the protein synthesis (Fig. 2B) [35]. The m.9176T>C (p.Leu217Pro) mutation was located in the highly conserved region between different species (Fig. 2C).

The mtDNA 4,977-bp deletion is one of the most frequently observed mtDNA mutations in human tissues, but neither patient showed the common deletion when long-template PCR was performed (Fig. 3). Although the whole-mtDNA sequencing

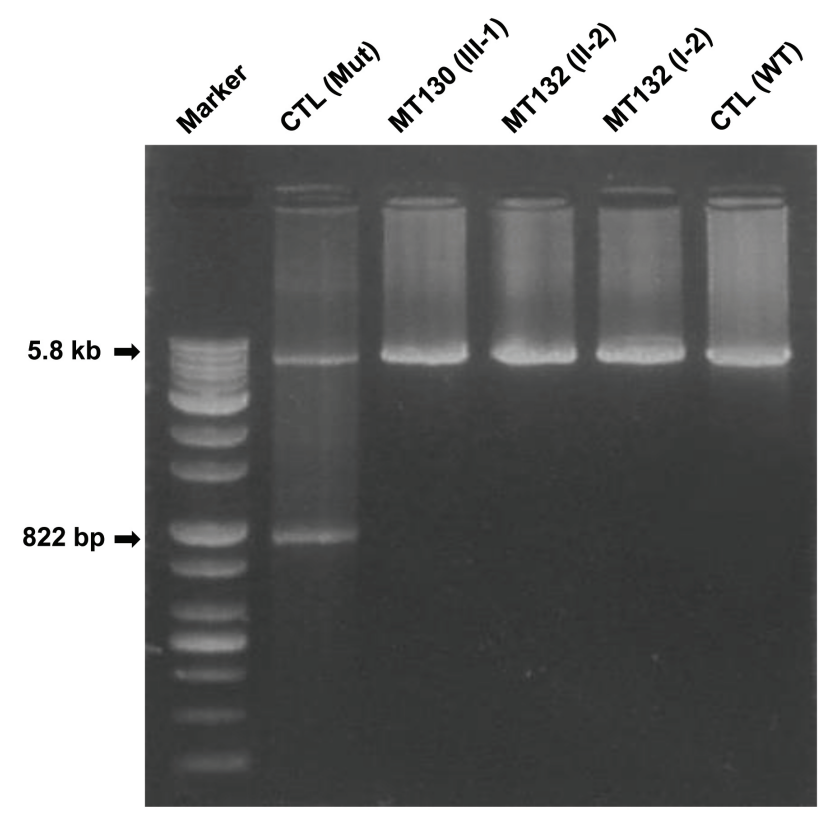

Fig. 3. Determination of the common mtDNA deletion (4,977 bp) by long-template polymerase chain reaction. The $5.8 \mathrm{~kb}$ and $822 \mathrm{bp}$ bands indicate the wild type and the common deletion type of mtDNA, respectively. Marker, $1 \mathrm{~kb}$ ladder; CTL (Mut), mtDNA mutant with 4,977 bp common deletion; CTL (WT), mtDNA wild type with no deletion. 
revealed many other variants, we considered them noncausative polymorphic variants because they were also observed in the healthy controls.

\section{Discussion}

The present study identified 2 mtDNA mutations ( $m .8344 \mathrm{~A}>\mathrm{G}$ in MT-TK and m.9176T>C [p.Leu217Pro] in MT-ATP6 from the MERRF patient and Leigh syndrome patient, respectively), by applying an mtDNA-targeted NGS tool. We believe that these mutations are the underlying cause of each disease phenotype, because both mutations have been reported to be the underlying causes of MERRF and Leigh syndromes [32-34] and no healthy control showed the same mutation. In addition, both mutations were located in the well-conserved protein region or functionally important pseudouridine arm of t-RNA.

The whole-mtDNA sequencing revealed several mtDNA variants that have been reported as polymorphisms as well as association (or secondary) factors in MITOMAP: these include m.3496G > T (more frequent in Japanese patients with Leber's hereditary optic neuropathy [LHON]) [36], m.4136A>G (a secondary genetic cause for LHON) [37], m.12026A>G (more frequent in Japanese diabetic patients) [38], and $\mathrm{m} .13708 \mathrm{G}>\mathrm{A}$ (a secondary genetic factor in Chinese LHON patients) [39]. In this study, because all these variants were also found in the healthy controls, we considered them as non-causative polymorphic variants. However, we could not completely exclude them as risk factors of the corresponding phenotypes.

Clinically, the patients with the m.9176T>C MT-ATP6 or m.8344A $>G$ mutations revealed typical clinical symptoms of Leigh syndrome and MERRF, respectively. Patient 1 afflicted with the Leigh syndrome had experienced neurological deterioration and psychomotor regression since 14 years of age. On brain $\mathrm{MRI}$, the symmetrical paramedian thalamus, brainstem, and periventricular area, which are hallmarks of the disease, were shown. Furthermore, patient 2 with the m.8344A $>G$ mutation showed progressive ataxia and myoclonus, which were compatible phenotypes with the MERRF.

Pathogenic mtDNA mutations are usually observed as a heteroplasmic state $[40,41]$. However, in this study, both causative mutations were observed in the affected probands as almost homoplasmic states. Moreover, the MERRF patient's unaffected mother (I-2 in MT132) showed a considerably high mutation load with an approximate rate of $80 \%$. It is known that the m.8344A>G mutation accounts for $80 \%$ to $90 \%$ of MERRF cases $[40,41]$. In spite of the high mutation load, the reason that the MERRF patient's mother was unaffected may be due to the DNA source used in this study. We used total DNA purified from the peripheral blood instead of affected tissues. The heteroplasmic state usually shows a tissue-specific rate. Recently, asymtomatic m.8344A $>\mathrm{G}$ carriers with high mutant loads have been reported [42].

The multi-organ involvement and overlapping symptoms make exact diagnosis of mitochondrial diseases difficult. Therefore, mutation screening is an important tool for the diagnosis of rare mitochondrial disorders. In this study, we applied mtDNA-targeted NGS to identify genetic causes of the 2 studied diseases. NGS technology has been recently introduced in mtDNA testing for mitochondrial diseases [28-30]. The application of NGS may reduce the testing cost and time, with high fidelity. In particular, heteroplasmic rates could be directly determined by counting altered reads from the total read depth at the corresponding mutation site. Therefore, we believe that whole-mtDNA sequencing by NGS will be a powerful tool for the molecular diagnosis of genetically and clinically heterogeneous mitochondrial diseases.

\section{References}

1. Zeviani M, Di Donato S. Mitochondrial disorders. Brain 2004;127: 2153-72

2. Zeviani M, Muntoni F, Savarese N, Serra G, Tiranti V, Carrara F, et al. A MERRF/MELAS overlap syndrome associated with a new point mutation in the mitochondrial DNA tRNA(Lys) gene. Eur J Hum Genet 1993;1:80-7.

3. Nakamura M, Nakano $S$, Goto $Y$, Ozawa M, Nagahama Y, Fukuyama $\mathrm{H}$, et al. A novel point mutation in the mitochondrial tRNA(Ser(UCN)) gene detected in a family with MERRF/MELAS overlap syndrome. Biochem Biophys Res Commun 1995;214:86-93.

4. Deschauer $M$, Müller $T_{1}$ Wieser $T$, Schulte-Mattler $W_{1}$ Kornhuber $M$, Zierz $\mathrm{S}$. Hearing impairment is common in various phenotypes of the mitochondrial DNA A3243G mutation. Arch Neurol 2001;58:1885-8.

5. Schmiedel J, Jackson S, Schäfer J, Reichmann H. Mitochondrial cytopathies. J Neurol 2003;250:267-77.

6. Shoffner JM, Lott MT, Lezza AM, Seibel P, Ballinger SW, Wallace DC. Myoclonic epilepsy and ragged-red fiber disease (MERRF) is associated with a mitochondrial DNA tRNA(Lys) mutation. Cell 1990; 61:931-7.

7. Yoneda M, Tanno Y, Horai S, Ozawa T, Miyatake T, Tsuji S. A common mitochondrial DNA mutation in the t-RNA(Lys) of patients with myoclonus epilepsy associated with ragged-red fibers. Biochem Int 1990;21:789-96. 
8. Berkovic SF, Shoubridge EA, Andermann F, Andermann E, Carpenter $S$, Karpati G. Clinical spectrum of mitochondrial DNA mutation at base pair 8344. Lancet 1991;338:457.

9. Santorelli FM, Mak SC, El-Schahawi M, Casali C, Shanske S, Baram TZ, et al. Maternally inherited cardiomyopathy and hearing loss associated with a novel mutation in the mitochondrial tRNA(Lys) gene (G8363A). Am J Hum Genet 1996;58:933-9.

10. Lertrit $P$, Noer AS, Byrne $E$, Marzuki S. Tissue segregation of a heteroplasmic mtDNA mutation in MERRF (myoclonic epilepsy with ragged red fibers) encephalomyopathy. Hum Genet 1992;90:251-4.

11. Yakubovskaya E, Mejia E, Byrnes J, Hambardjieva E, Garcia-Diaz M. Helix unwinding and base flipping enable human MTERF1 to terminate mitochondrial transcription. Cell 2010;141:982-93.

12. Melone MA, Tessa A, Petrini $S$, Lus G, Sampaolo S, di Fede G, et al. Revelation of a new mitochondrial DNA mutation (G12147A) in a MELAS/MERFF phenotype. Arch Neurol 2004;61:269-72.

13. Mancuso M, Filosto M, Mootha VK, Rocchi A, Pistolesi S, Murri L, et al. A novel mitochondrial tRNAPhe mutation causes MERRF syndrome. Neurology 2004;62:2119-21.

14. Naini $A B$, Lu J, Kaufmann P, Bernstein RA, Mancuso M, Bonilla $E$, et al. Novel mitochondrial DNA ND5 mutation in a patient with clinical features of MELAS and MERRF. Arch Neurol 2005;62:473-6.

15. Leigh D. Subacute necrotizing encephalomyelopathy in an infant. J Neurol Neurosurg Psychiatry 1951;14:216-21.

16. Dahl HH. Getting to the nucleus of mitochondrial disorders: identification of respiratory chain-enzyme genes causing Leigh syndrome. Am J Hum Genet 1998;63:1594-7.

17. DiMauro S, De Vivo DC. Genetic heterogeneity in Leigh syndrome. Ann Neurol 1996;40:5-7.

18. Rahman S, Blok RB, Dahl HH, Danks DM, Kirby DM, Chow CW, et al. Leigh syndrome: clinical features and biochemical and DNA abnormalities. Ann Neurol 1996;39:343-51.

19. Goto Y, Nonaka I, Horai S. A new mtDNA mutation associated with mitochondrial myopathy, encephalopathy, lactic acidosis and strokelike episodes (MELAS). Biochim Biophys Acta 1991;1097:238-40.

20. Silvestri G, Moraes CT, Shanske S, Oh SJ, DiMauro S. A new mtDNA mutation in the tRNA(Lys) gene associated with myoclonic epilepsy and ragged-red fibers (MERRF). Am J Hum Genet 1992;51:1213-7.

21. Sweeney MG, Bundey $S$, Brockington $M$, Poulton KR, Winer JB, Harding AE. Mitochondrial myopathy associated with sudden death in young adults and a novel mutation in the mitochondrial DNA leucine transfer RNA(UUR) gene. O J Med 1993;86:709-13.

22. Chinnery PF, Brown DT, Andrews RM, Singh-Kler R, Riordan-Eva $P$, Lindley $J$, et al. The mitochondrial ND6 gene is a hot spot for mutations that cause Leber's hereditary optic neuropathy. Brain $2001 ; 124: 209-18$.
23. Crimi M, Papadimitriou A, Galbiati $S$, Palamidou P, Fortunato $F$, Bordoni $A$, et al. A new mitochondrial DNA mutation in ND3 gene causing severe Leigh syndrome with early lethality. Pediatr Res 2004; 55:842-6.

24. Anderson $\mathrm{S}$, Bankier AT, Barrell BG, de Bruijn MH, Coulson AR, Drouin $J$, et al. Sequence and organization of the human mitochondrial genome. Nature 1981;290:457-65.

25. Kwon SJ, Park SS, Kim JM, Ahn TB, Kim SH, Kim J, et al. Investigation of common mitochondrial point mutations in Korea. Ann N Y Acad Sci 2004;1011:339-44.

26. Choi BO, Hwang JH, Kim J, Cho EM, Cho SY, Hwang SJ, et al. A MELAS syndrome family harboring two mutations in mitochondrial genome. Exp Mol Med 2008:40:354-60.

27. Choi BO, Hwang JH, Cho EM, Jeong EH, Hyun YS, Jeon HJ, et al. Mutational analysis of whole mitochondrial DNA in patients with MELAS and MERRF diseases. Exp Mol Med 2010;42:446-55.

28. Carroll CJ, Brilhante V, Suomalainen A. Next-generation sequencing for mitochondrial disorders. Br J Pharmacol 2014;171:1837-53.

29. Vellarikkal SK, Dhiman H, Joshi K, Hasija Y, Sivasubbu S, Scaria V. mit-o-matic: a comprehensive computational pipeline for clinical evaluation of mitochondrial variations from next-generation sequencing datasets. Hum Mutat 2015;36:419-24.

30. Calabrese $C$, Simone $D$, Diroma MA, Santorsola $M$, Guttà $C$, Gasparre G, et al. MToolBox: a highly automated pipeline for heteroplasmy annotation and prioritization analysis of human mitochondrial variants in high-throughput sequencing. Bioinformatics 2014;30: 3115-7.

31. Andrews RM, Kubacka I, Chinnery PF, Lightowlers RN, Turnbull DM, Howell N. Reanalysis and revision of the Cambridge reference sequence for human mitochondrial DNA. Nat Genet 1999;23:147.

32. Shoffner JM, Bialer MG, Pavlakis SG, Lott M, Kaufman A, Dixon J, et al. Mitochondrial encephalomyopathy associated with a single nucleotide pair deletion in the mitochondrial tRNALeu(UUR) gene. Neurology 1995;45:286-92.

33. Campos $Y$, Martín MA, Rubio JC, Solana LG, Garcia-Benayas $C$, Terradas JL, et al. Leigh syndrome associated with the T9176C mutation in the ATPase 6 gene of mitochondrial DNA. Neurology 1997:49:595-7.

34. Ronchi $D$, Bordoni $A$, Cosi $A$, Rizzuti $M$, Fassone $E_{1}$ Di Fonzo $A$, et al. Unusual adult-onset Leigh syndrome presentation due to the mitochondrial m.9176T>C mutation. Biochem Biophys Res Commun 2011;412:245-8.

35. Noer AS, Sudoyo $H$, Lertrit $P$, Thyagarajan D, Utthanaphol P, Kapsa $R$, et al. A tRNA(Lys) mutation in the mtDNA is the causal genetic lesion underlying myoclonic epilepsy and ragged-red fiber (MERRF) syndrome. Am J Hum Genet 1991;49:715-22. 
36. Matsumoto $M$, Hayasaka $S$, Kadoi $C$, Hotta $Y$, Fujiki $K_{1}$ Fujimaki $T_{\text {, et }}$ al. Secondary mutations of mitochondrial DNA in Japanese patients with Leber's hereditary optic neuropathy. Ophthalmic Genet 1999;20: 153-60.

37. Obermaier-Kusser B, Lorenz B, Schubring S, Paprotta A, Zerres K, Meitinger $T$, et al. Features of mtDNA mutation patterns in European pedigrees and sporadic cases with Leber hereditary optic neuropathy. Am J Hum Genet 1994;55:1063-6.

38. Tawata $M$, Ohtaka $M$, Iwase $E$, Ikegishi $Y$, Aida $K$, Onaya T. New mitochondrial DNA homoplasmic mutations associated with Japanese patients with type 2 diabetes. Diabetes 1998;47:276-7.

39. Wang HW, Jia $X$, Ji $Y$, Kong $Q P$, Zhang $Q$, Yao $Y G$, et al. Strikingly different penetrance of LHON in two Chinese families with primary mutation G11778A is independent of mtDNA haplogroup background and secondary mutation G13708A. Mutat Res 2008;643:48-53.

40. Spyropoulos A, Manford M, Horvath R, Alston CL, Yu-Wai-Man P, He $L$, et al. Near-identical segregation of mtDNA heteroplasmy in blood, muscle, urinary epithelium, and hair follicles in twins with optic atrophy, ptosis, and intractable epilepsy. JAMA Neurol 2013;70:15525.

41. Pallotti $F$, Binelli $G$, Fabbri $R$, Valentino $M L$, Vicenti $R$, Macciocca $M$, et al. A wide range of $3243 \mathrm{~A}>\mathrm{G} / \mathrm{tRNALeu}(\mathrm{UUR})$ (MELAS) mutation loads may segregate in offspring through the female germline bottleneck. PLoS One 2014;9:e96663.

42. Mancuso M, Orsucci D, Angelini C, Bertini E, Carelli V, Comi GP, et al. Phenotypic heterogeneity of the $8344 \mathrm{~A}>\mathrm{G}$ mtDNA "MERRF" mutation. Neurology 2013;80:2049-54. 